\title{
Survival and prognostic factors in patients undergoing extended pulmonary metastasectomy
}

\author{
YO KAWAGUCHI $^{1,2}$, JUN HANAOKA ${ }^{1}$, YASUHIKO OHSHIO ${ }^{1}$, KEIGO OKAMOTO $^{1}$, \\ RYOSUKE KAKU $^{1}$, KAZUKI HAYASHI ${ }^{1}$, TAKUYA SHIRATORI ${ }^{1}$ and MAKOTO YODEN ${ }^{1}$ \\ ${ }^{1}$ Division of General Thoracic Surgery, Department of Surgery, Shiga University of Medical Science, Otsu, Shiga 520-2192; \\ ${ }^{2}$ Department of General Thoracic Surgery, Kusatsu General Hospital, Kusatsu, Shiga 525-8585, Japan
}

Received February 19, 2020; Accepted July 15, 2020

DOI: $10.3892 / \operatorname{mco} .2020 .2118$

\begin{abstract}
Extended pulmonary metastasectomy has become feasible in patients with extrapulmonary metastases and multiple or bilateral pulmonary metastases. Even peritoneal dissemination is considered to be curable in modern medicine. Therefore, it is necessary to analyze the prognosis of patients undergoing complete pulmonary metastasectomy. A total of 80 patients who underwent pulmonary resection for lung metastases were retrospectively analyzed. The eligibility criteria for the present study were as follows: i) the primary tumor was controlled; ii) if extrapulmonary metastases (including peritoneal dissemination) existed, these were controlled by local treatment or such treatment was planned; iii) the one to three months follow-up computed tomography (CT) following the first assessment revealed no increase of pulmonary metastatic disease; iv) pulmonary metastases could be resected completely. The overall 5- and 10-year survival rates were 71.7 and $41.5 \%$, respectively. Applying the extended criteria for surgery, the present study demonstrated that pulmonary metastasectomy resulted in a good patient prognosis.
\end{abstract}

\section{Introduction}

Despite the developments in the chemo- and radiotherapy, and treatment with biological agents of patients with pulmonary metastases, surgery is still an important option in this situation. Thomford et al (1), presented the criteria for pulmonary metastasectomy, which included adequate cardiopulmonary reserves of the patient, the control of the primary tumor site, no evidence of metastatic disease elsewhere in the body and that the pulmonary metastases being limited to one lung.

Correspondence to: Dr Yo Kawaguchi, Division of General Thoracic Surgery, Department of Surgery, Shiga University of Medical Science, Tsukinowacho, Seta, Otsu, Shiga 520-2192, Japan E-mail: kawaguchi1228@yahoo.co.jp

Key words: pulmonary metastases, metastasectomy, pulmonary resection, peritoneal dissemination, survival
However, these criteria have been continuously extended and modified over time. Today, extended pulmonary metastasectomy has become feasible in patients with distant metastases such as a solitary liver metastasis or multiple and bilateral pulmonary metastases. Therefore, it is time to analyze the survival of patients undergoing extended pulmonary metastasectomy.

Peritoneal dissemination originating from gastrointestinal tract organs, pseudomyxoma peritonei, or ovarian cancer has for long been considered as a lethal disease with poor prognosis. Recently, however, new treatment methods have emerged, and nowadays even cure can be achieved with cytoreductive surgery and hyperthermic intraperitoneal chemotherapy in these patients (2). Consequently, we also need to analyze the prognosis of patients with peritoneal dissemination controlled by the treatments.

The purpose of this study was to analyze the current data for overall survival after extended pulmonary metastasectomy, including in patients with treated peritoneal dissemination at our institution and to clarify whether above described prognostic factors affect survival.

\section{Patients and methods}

We obtained institutional review board approval for this study (approval no. 2019-015). The requirement for informed patient consent was waived because of the retrospective nature of the study.

The present study was a retrospective analysis of the medical records of 80 patients who underwent complete resection for lung metastases at our hospital between September 2007 and February 2019. The record of each patient was reviewed for age, sex, primary tumor, disease-free interval, number of pulmonary nodules, side of pulmonary nodules, extrapulmonary metastases, the maximum diameter of the pulmonary nodules, peritoneal dissemination of the primary tumor and surgical procedure for metastasectomy. In case multiple metastases were present, the diameter of the largest nodule was recorded.

The eligibility criteria for this study were as follows: i) the primary tumor was controlled; ii) if extrapulmonary metastases (including peritoneal dissemination) existed, these had been controlled by local treatment or such treatment was 
planned; iii) the one to three months follow-up computed tomography (CT) after the first assessment revealed no increase of pulmonary metastatic disease and iv) pulmonary metastases could be resected completely.

We excluded patients whose performance status was over 2 , or whose forced expiratory volume in one second during the preoperative pulmonary function test was lower than $1,000 \mathrm{ml}$. We included any types of primary tumors and did not exclude patients with multiple or bilateral pulmonary metastases. All patients with peritoneal dissemination received cytoreductive surgery and hyperthermic intraperitoneal chemotherapy prior to pulmonary resection of metastases. We judged extrapulmonary metastases as 'controlled' when one to six months follow-up imaging examinations revealed no evidence of relapse after local treatment. If a more effective treatment modality than surgery was available, the patients received the optimal treatment.

Our standard surgical method includes the complete resection of nodules after palpation, while mediastinal lymph node dissection is not routinely performed. The surgical approach is performed by thoracoscopically assisted or lateral thoracotomy. We usually attempt to remove pulmonary nodules through a wedge resection whereas hilar nodules require lobectomy or pneumonectomy. We defined 'complete resection' as no obvious residual cancer on the resection stump of the lung. Patients are followed-up for up to 10 years after metastasectomy.

Statistical analysis was performed using SPSS Statistics for Windows, v22 (SPSS Inc.). The Kaplan-Meier method was used to determine overall, relapse-free, and median survival from the time of metastasectomy to the last follow-up, death or relapse. The log-rank test was used to compare survival differences for each variable. $\mathrm{P}<0.05$ was considered to indicate a statistically significant difference.

\section{Results}

During the study period, 80 patients underwent pulmonary resection for lung metastases in our hospital. We extracted the data of these 80 patients. Their clinical, tumor and surgical characteristics are shown in Table I. The mean age at the time of thoracic surgery was 63.1 years, range 29-84 years. There were 46 male and 34 female patients. The mean period between primary tumor surgery and pulmonary resection was 39.7 months. The primary tumor was colorectal cancer in 43 patients, pseudomyxoma peritonei in 9 patients, head and neck cancer in 8 patients and other cancers in 20 patients. Twenty-two patients were treated for peritoneal dissemination and were recurrence-free based on computed tomography or magnetic resonance imaging during a follow-up of at least eight months after the intervention. The primary tumor of peritoneal dissemination was colorectal cancer in 11 patients, pseudomyxoma peritonei in 9 patients and uterine leiomyosarcoma in 2 patients. Fifty-five patients received wedge resection, 13 patients received segmentectomy, 10 patients received lobectomy, and 2 patients received pneumonectomy. Post-operative complications occurred in 13 patients (16.3\%), which consisted of pneumonia in 3 patients, empyema in 3 patients, intrathoracic bleeding in 1 patient, gastrointestinal bleeding in 1 patient, respiratory failure requiring oxygen
Table I. Characteristics of 80 patients undergoing pulmonary resection for lung metastases.

\begin{tabular}{|c|c|c|}
\hline Characteristics & $\begin{array}{l}\text { Number of patients } \\
n=80\end{array}$ & $\%$ \\
\hline Age (years) & $63.1(29-84)$ & - \\
\hline \multicolumn{3}{|l|}{ Sex } \\
\hline Male & 46 & 58 \\
\hline Female & 34 & 42 \\
\hline \multicolumn{3}{|l|}{ Primary tumor } \\
\hline Colorectal & 43 & 54 \\
\hline Pseudomyxoma peritonei & 9 & 11 \\
\hline Head and neck cancer & 8 & 10 \\
\hline Other cancers & 20 & 25 \\
\hline Disease-free interval (Mo) & $26.2(0-156)$ & - \\
\hline Pulmonary metastases (No) & $1.7(1-13)$ & \\
\hline 1 & 58 & 73 \\
\hline 2 & 11 & 14 \\
\hline$\geq 3$ & 11 & 14 \\
\hline \multicolumn{3}{|l|}{ Side } \\
\hline Unilateral & 66 & 82 \\
\hline Bilateral & 14 & 18 \\
\hline \multicolumn{3}{|l|}{ Extrapulmonary metastasis } \\
\hline Yes & 13 & 16 \\
\hline No & 67 & 84 \\
\hline Maximum tumor size & $20.8(7-146)$ & \\
\hline$\leq 20 \mathrm{~mm}$ & 55 & 69 \\
\hline$>20 \mathrm{~mm}$ & 25 & 31 \\
\hline \multicolumn{3}{|l|}{ Peritoneal dissemination } \\
\hline Yes & 22 & 28 \\
\hline No & 58 & 72 \\
\hline \multicolumn{3}{|l|}{ Surgical procedure } \\
\hline Wedge resection & 55 & 69 \\
\hline Segmentectomy & 13 & 16 \\
\hline Lobectomy & 10 & 13 \\
\hline Pneumonectomy & 2 & 3 \\
\hline
\end{tabular}

inhalation in 1 patient, heart failure in 1 patient, ileus in 1 patient, deterioration in liver function in 1 patient and recurrent nerve paralysis in 1 patient.

The mean follow-up after thoracic surgery was 36 months, range 1-120 months. The 5-year and 10-year survival rates after metastasectomy were 71.7 and $41.5 \%$, respectively (Fig. 1). Relapse-free survival at five years after metastasectomy was $33.6 \%$ (Fig. 1) and median survival time 84.7 months.

We compared the median survival times with the clinical factors of age, gender, primary tumor, disease-free interval, number of pulmonary metastases, laterality of the metastases, maximum tumor size, extrapulmonary metastases, and peritoneal dissemination. In the univariate analysis, none of these factors showed a significant association with the median survival time (Table II). However, bilateral metastases, extrapulmonary metastases, treated peritoneal dissemination, 

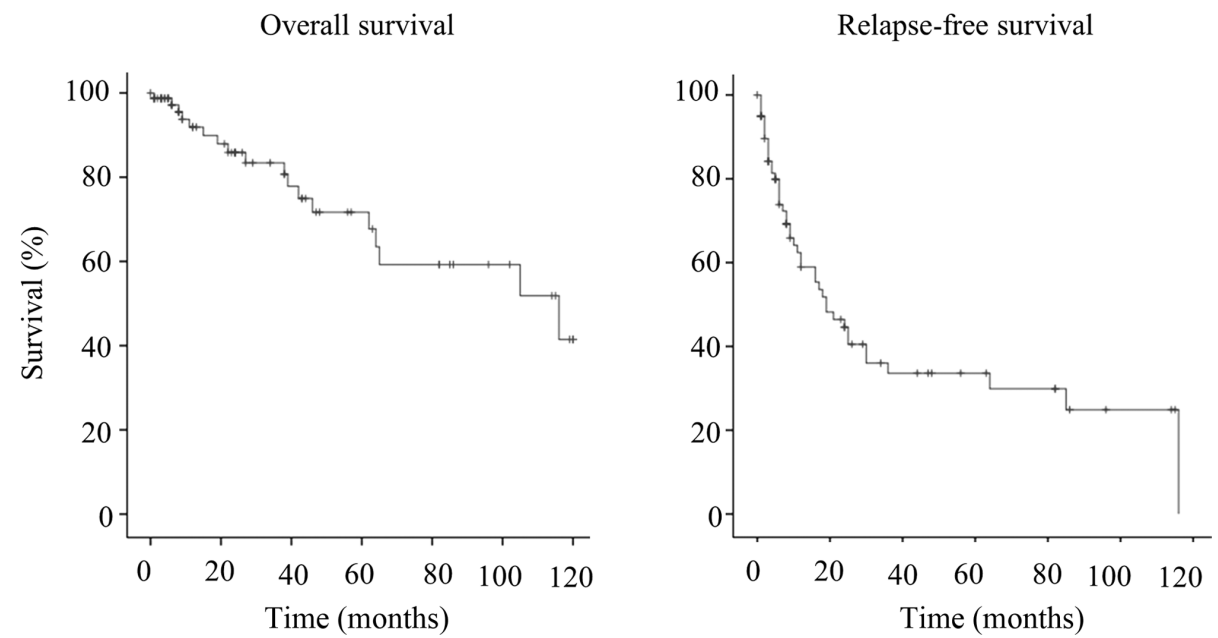

Figure 1. Kaplan-Meier curves for overall and relapse-free survival in 80 patients undergoing pulmonary resection for lung metastases.

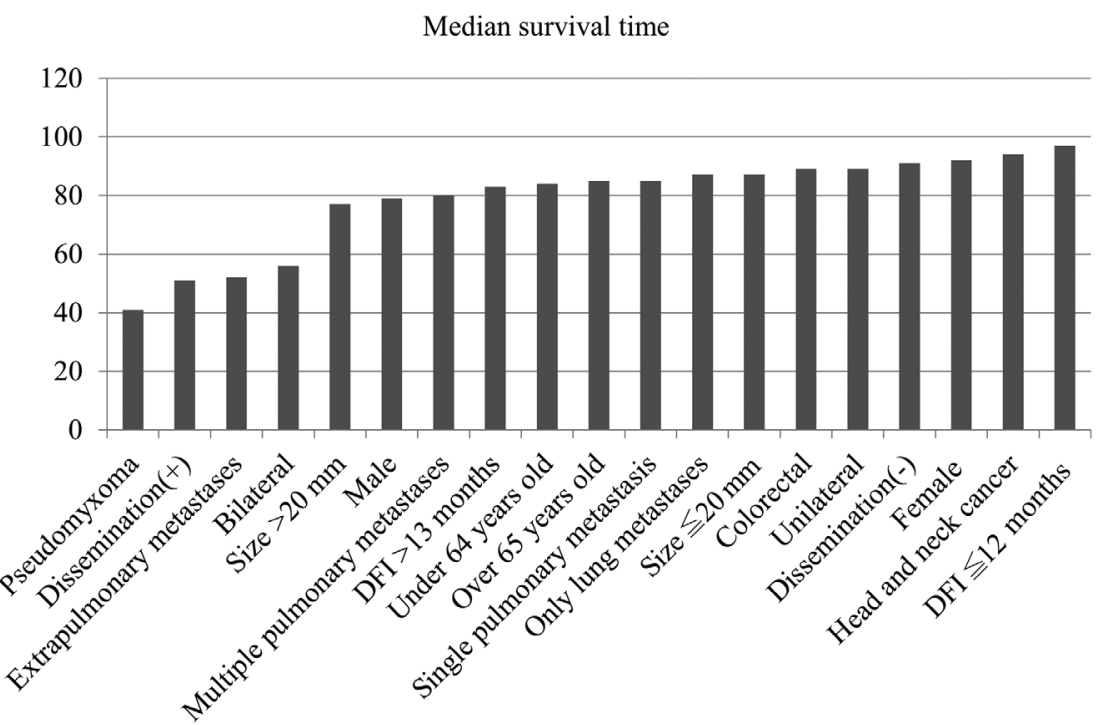

Figure 2. Median survival time in relation to clinical factors in 80 patients undergoing pulmonary resection for lung metastases. DFI, disease-free interval.

and histology of pseudomyxoma peritonei tended to be associated with lower survival (Fig. 2).

There was no significant difference $(\mathrm{P}=0.059)$ in the 5 -year survival of patients with peritoneal dissemination $(55.8 \%)$ and patients without history of peritoneal dissemination (76.4\%) (Fig. 3).

\section{Discussion}

The indication for pulmonary metastasectomy has been based on Thomford's criteria (1) for a long time, but recently, it was increasingly extended to patients with multiple lesions, bilateral lesions, and solitary liver metastasis. Furthermore, the development of cytoreductive surgery and hyperthermic intraperitoneal chemotherapy appears to contribute to better prospects of cure for patients with peritoneal dissemination (2). In this study, we included survival in patients who had been or were foreseen to be treated for peritoneal dissemination.

At our institution, the extended criteria for pulmonary resection include multiple lesions, extrapulmonary metastases, bilateral metastases, and peritoneal dissemination. A previous article reported that multiple pulmonary metastases were not a prognostic factor for overall survival (3) whereas another article reported them to be a significant factor (4). In our study, the prognosis of multiple pulmonary metastases was no different from a solitary metastasis. Although patients with bilateral metastases, extrapulmonary metastases, and peritoneal dissemination tended to obtain less benefit from surgery, the differences between their mean survival time and those of other patients were not statistically significant. Several studies have investigated the difference between unilateral and bilateral metastases in terms of survival. Only two articles reported that unilateral pulmonary metastases were a positive prognostic factor for overall survival $(3,5)$. There have been several reports about metastasectomy in patients with extrapulmonary metastases. McAfee et al (6) and Saito et al (7), both showed that the presence of resectable or controllable extrapulmonary metastases before or at the time of thoracotomy was not associated with decreased survival. These results suggest that metastasectomy might be beneficial 
Table II. Median survival time in relation to clinical factors in 80 patients undergoing pulmonary resection for lung metastases.

\begin{tabular}{|c|c|c|c|}
\hline Variables & $\mathrm{N}$ & $\begin{array}{c}\text { Mean } \\
\text { survival } \\
\text { time } \\
\text { (months) }\end{array}$ & P-value \\
\hline Age (years) & & & 0.700 \\
\hline$<65$ & 38 & 84.7 & \\
\hline$\geq 65$ & 42 & 84.1 & \\
\hline Sex & & & 0.379 \\
\hline Male & 46 & 79.1 & \\
\hline Female & 34 & 92.5 & \\
\hline \multicolumn{4}{|l|}{ Primary tumor } \\
\hline Colorectal & 43 & 81.1 & 0.686 \\
\hline Pseudomyxoma peritonei & 9 & 41.1 & 0.296 \\
\hline Head and neck cancer & 8 & 94.4 & 0.277 \\
\hline Disease-free interval (Months) & & & 0.821 \\
\hline $0-12$ & 30 & 96.6 & \\
\hline $13-$ & 48 & 83.0 & \\
\hline Pulmonary metastases (No) & & & 0.891 \\
\hline 1 & 58 & 84.6 & \\
\hline$\geq 2$ & 22 & 80.0 & \\
\hline Laterality of the metastases & & & 0.124 \\
\hline Unilateral & 66 & 88.9 & \\
\hline Bilateral & 14 & 56.4 & \\
\hline Maximum tumor size & & & 0.513 \\
\hline$\leq 20 \mathrm{~mm}$ & 55 & 87.0 & \\
\hline$>20 \mathrm{~mm}$ & 25 & 77.1 & \\
\hline Extrapulmonary metastases & & & 0.261 \\
\hline Yes & 13 & 52.0 & \\
\hline No & 67 & 87.3 & \\
\hline Peritoneal dissemination & & & 0.059 \\
\hline Yes & 22 & 50.9 & \\
\hline No & 58 & 90.9 & \\
\hline
\end{tabular}

when extrapulmonary metastases are controllable, for example, in the case of a solitary liver metastasis.

The original aspect of our study was to include patients with previously treated peritoneal dissemination. There have been no reports on pulmonary metastasectomy in these patients. Peritoneal dissemination originating from the gastrointestinal organs, pseudomyxoma peritonei or ovarian cancer has often been considered as a systemic and lethal disease with poor prognosis. As a consequence, supportive care and systemic chemotherapy were the main treatment strategy for long, even though systemic chemotherapy is not sufficient to control peritoneal dissemination. However, more recently, specialized hospitals have reported that even cure could be achieved by the combination of cytoreductive surgery and hyperthermic intraperitoneal chemotherapy, with 5-year survival rates of $45 \%$ in colorectal cancer, $10.7 \%$

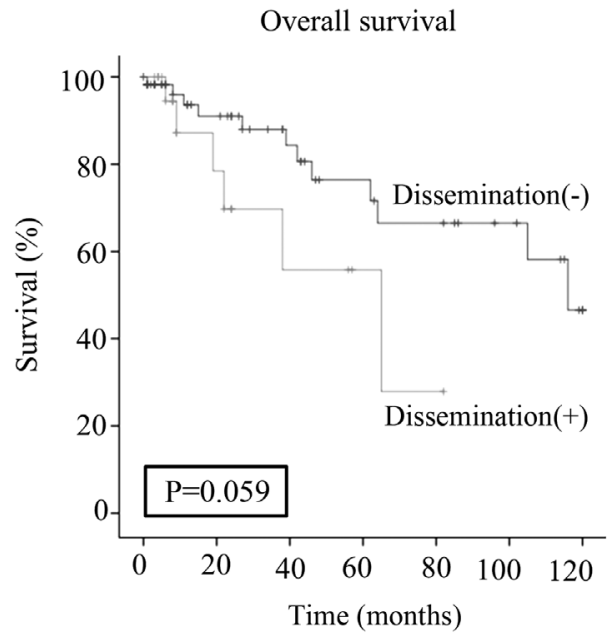

Figure 3. Overall survival after pulmonary metastasectomy in 80 patients with cancer with $(n=22)$ and without $(n=58)$ peritoneal dissemination.

in gastric cancer, $58 \%$ in ovarian cancer and a 10 -year survival rate of $63 \%$ in pseudomyxoma peritonei (2) after cytoreductive surgery. Furthermore, we showed for the first time a 5-year survival rate of $55.8 \%$ and a mean survival time of 50.9 months after pulmonary metastasectomy in these patients. Considering that there was long period between primary tumor surgery and pulmonary metastasectomy (39.7 months) in our study, our survival rate after pulmonary metastasectomy might be sufficiently acceptable. With increased survival in patients with peritoneal dissemination, the opportunity of pulmonary metastasectomy will also increase.

With regard to the different primary tumors, colorectal and head and neck cancer achieved a mean survival time of 81.1 and 94.4 months, respectively, whereas pseudomyxoma peritonei showed a poor prognosis. Pseudomyxoma peritonei is divided into a low-grade and high-grade form. The 5-year overall survival rate was found to be $63 \%$ for low grade but only $23 \%$ for high grade disease after surgical resection $(8,9)$. Metastases tend to occur in high-grade disease, which might contribute to the poor prognosis of pulmonary metastases of pseudomyxoma peritonei in our study.

The overall 5-year and 10-year survival rate in our study was 71.7 and $41.5 \%$, respectively, and the mean survival time was 84.7 months. Thomford et al reported a 5-year survival rate of $30.3 \%$. Another more recent report in 2012 showed a 5 -year survival rate of $43.7 \%$ and a 10 -year survival rate of about $30 \%$ (4). Despite our extended criteria for pulmonary resection of metastases, we achieved better survival rates. The reason for the better survival might be threefold. First, the new modes of non-surgical treatment such as systemic chemotherapy, hyperthermic intraperitoneal chemotherapy, biological agents and radiotherapy might prolong the overall survival in patients with advanced cancer. Second, we excluded all cases with increasing pulmonary metastases during one to three months. We hypothesized that when metastasic disease increased in the short term, systemic chemotherapy was likely to be more effective than local treatment such as surgery. Third, we extracted data only on cases with complete resection of pulmonary 
metastases. Predictors of good prognosis usually include long disease-free interval, the histology of the primary tumor, a small number of metastases, unilateral metastases, and complete resection. Among these, the most consistent predictor is complete resection (10).

The main limitation of this study is its retrospective nature. The final decision to select a patient for surgery may vary among surgeons. Therefore, our study does not reflect the entire population of patients with pulmonary metastases. A second limitation is the small number of cases. This might result in insufficient power to detect significant differences, even in cases that potentially might show statistical differences. The final limitation is the heterogeneity of the histology of the primary tumor. Tumor characteristics such as growth speed, metastatic potential, and anticancer drug sensitivity differ depending on the histology of the primary lesion. We also recognize that non-surgical treatment such as chemotherapy, thermotherapy, biological agents or radiotherapy may substantially affect the prognosis of patients in our study. Based on this pilot study, we plan to perform a prospectively designed study with sufficiently large patient numbers and segregation by histology.

In conclusion, our extended pulmonary metastasectomy achieved 5-year and 10-year survival rates of 71.7 and $41.5 \%$, respectively. Extended criteria in our study including multiple lung lesions, bilateral metastases, controlled extrapulmonary metastases and controlled peritoneal dissemination might be acceptable for pulmonary metastasectomy.

\section{Acknowledgements}

Not applicable.

\section{Funding}

No funding was received.

\section{Availability of data and materials}

The datasets used and/or analyzed during the present study are available from the corresponding author on reasonable request.

\section{Authors' contributions}

YK has made substantial contributions to conception, design, acquisition of data, analysis and interpretation of data, and been involved in drafting the manuscript and revising it critically for important intellectual content. JH, YO, KO, RK, KH, TS and MY made substantial contributions to analysis and interpretation of data. All authors reviewed and approved the final manuscript.

\section{Ethics approval and consent to participate}

Institutional review board approval was obtained for the current study (approval no. 2019-015). The requirement for informed patient consent was waived because of the retrospective nature of the study.

\section{Patient consent for publication}

Not applicable.

\section{Competing interests}

The authors have no competing interests.

\section{References}

1. Thomford NR, Woolner LB and Clagett OT: The surgical treatment of metastatic tumors in the lungs. J Thorac Cardiovasc Surg 49: 357-363, 1965.

2. Canbay E, Torun BC, Torun ES and Yonemura Y: Evolution of management in peritoneal surface malignancies. Ulus Cerrahi Derg 32: 203-207, 2016.

3. Younes RN, Abrao F and Gross J: Pulmonary metastasectomy for colorectal cancer: Long-Term survival and prognostic factors. Int J Surg 11: 244-248, 2013.

4. Younes RN, Fares AL and Gross JL: Pulmonary metastasectomy: A multivariate analysis of 440 patients undergoing complete resection. Interact Cardiovasc Thorac Surg 14: 156-161, 2012.

5. Chen F, Hanaoka N, Sato K, Fujinaga T, Sonobe M, Shoji T, Sakai H, Miyahara R, Bando T, Okubo K, et al: Prognostic factors of pulmonary metastasectomy for colorectal carcinomas. World J Surg 33: 505-511, 2009.

6. McAfee MK, Allen MS, Trastek VF, Ilstrup DM, Deschamps C and Pairolero PC: Colorectal lung metastases: Results of surgical excision. Ann Thorac Surg 53: 780-785, 1992.

7. Saito Y, Omiya H, Kohno K, Kobayashi T, Itoi K, Teramachi M, Sasaki M, Suzuki H, Takao H and Nakade M: Pulmonary metastasectomy for 165 patients with colorectal carcinoma: A prognostic assessment. J Thorac Cardiovasc Surg 124: 1007-1013, 2002.

8. Carr NJ, Finch J, Ilesley IC, Chandrakumaran K, Mohamed F, Mirnezami A, Cecil T and Moran B: Pathology and prognosis in pseudomyxoma peritonei: A review of 274 cases. J Clin Pathol 65: 919-923, 2012.

9. Kawaguchi Y, Hanaoka J, Ohshio Y, Okamoto K, Kaku R, Hayashi K, Shiratori T and Yoden M: Patient survival after surgical management in intrathoracic pseudomyxoma peritonei. Ann Surg Oncol 26: 238-243, 2019.

10. Pastorino U, Buyse M, Friedel G, Ginsberg RJ, Girard P, Goldstraw P, Johnston M, McCormack P, Pass H and Putnam JB Jr; International Registry of Lung Metastases: Long-Term results of lung metastasectomy: Prognostic analyses based on 5206 cases. J Thorac Cardiovasc Surg 113: 37-49, 1997. 